\title{
La dimensión urbana de las centralidades de Lima Norte: cambios y permanencias en la estructura metropolitana
}

Pablo Vega-Centeno. Pontificia Universidad Católica del Perú, Lima, Perú.

RESUMEN | En el proceso de reestructuración urbana que Lima experimenta desde fines del siglo $\mathrm{xx}$, varios autores peruanos destacan la emergencia de nuevos centros metropolitanos en lo que fueron antiguas periferias urbanas, en particular en la zona norte. Examinando la dimensión urbana de estos centros a través de su importancia como destino laboral o de abastecimiento, se observa que aún desempeñan un rol secundario dentro de la estructura metropolitana; asimismo, las percepciones de sus usuarios con relación a diferentes características de su dimensión urbana tienden a ser negativas. En suma, si bien estos llamados nuevos centros cumplen con satisfacer necesidades cotidianas a su importante entorno residencial, principalmente en materia de abastecimiento, todavía distan de erigirse como centralidades metropolitanas.

PALABRAS CLAVE | centros urbanos, espacio público, estructura urbana.

ABSTRACT | Regarding the urban restructuration process undergoing in Lima since the late 20th century, some Peruvian authors emphasize the emergence of major metropolitan centers in old urban peripheries such as the northern side of Lima. Considering the urban dimensions of these centers, as important employment and provision destinations, we can notice that they still have a secondary function in the metropolitan structure. Also, citizen perceptions about different characteristics of their urban dimensions tend to be negative. In summary, although these new centers satisfy several daily urban necessities for their residential surroundings, especially in terms of provision, they are still far from becoming major metropolitan centers.

KEYWORDs | urban centers, public space, urban structure. 


\section{Introducción}

Lima, al igual que otras ciudades latinoamericanas, se convirtió repentinamente en metrópoli como consecuencia del crecimiento demográfico explosivo que muchas experimentaron, y que en el Perú ocurrió principalmente entre 1940 y 1980. Este proceso obedeció de manera importante a un fenómeno de migraciones internas hacia las ciudades por parte de población de origen rural, que se trasladaba por la falta de oportunidades de subsistencia en un campo sobrepoblado. Si bien el incremento poblacional no se ha detenido en estos primeros lustros del siglo xxI, la intensidad del proceso demográfico sí ha disminuido. En cambio, el crecimiento vertical de las edificaciones experimenta un importante impulso, lo que augura una ocupación más densa del espacio para las próximas décadas. La expansión urbana a través de barriadas ya no es el fenómeno excluyente de la producción del espacio en ciudades como Lima; las múltiples intervenciones del capital inmobiliario en productos ofrecidos tanto dentro del casco urbano como en la periferia, al igual que la densificación de las antiguas barriadas, constituyen tipos de crecimiento urbano tanto o más importantes que el anterior. Y si bien existen cambios en las formas de ocupación residencial, es poco lo que sabemos en relación con aquellos espacios urbanos que concentran oportunidades laborales y actividades comerciales, entre otros aspectos.

En Lima, el centro histórico dejó de ser el único gran centro de la ciudad hacia 1980, surgiendo concentraciones importantes como Miraflores, pero sin dejar de ocupar la gran área central de la ciudad. Entrando en el siglo XXI, algunos autores destacan el desarrollo de nuevas centralidades metropolitanas, esta vez fuera del área central de Lima, en las grandes zonas urbanas denominadas Lima Norte, Lima Este, Lima Sur o Callao.

Las primeras décadas del siglo XXI encuentran a Lima posicionada como una urbe atractiva para las inversiones económicas en América Latina. Ello supone que existen centros o nodos importantes, sea por las especializaciones que concentran o por el movimiento de capitales que atraen. Pero cómo forman parte del tejido de la ciudad y qué importancia tienen para la vida cotidiana de sus habitantes, son aspectos necesarios de considerar.

En este contexto de reestructuración urbana, interesa conocer la magnitud que cobran estos nuevos centros localizados fuera del área central como destino cotidiano de los limeńos, y las percepciones que existen respecto de ellos.

\section{Centralidades en la estructura urbana, entre nodos y lugares}

En este artículo, el objeto de estudio está constituido por los centros urbanos en tanto concentraciones de actividades urbanas que atraen un flujo de habitantes, vehículos y bienes (Polydorides, 1983, p. 1). Principalmente se alude a actividades económicas, pero también se los vincula a servicios públicos y equipamientos que acogen diferentes actividades sociales y culturales. En nuestro caso, interesa conocer la dimensión urbana de estos centros, entendiendo lo urbano como la vida social que se produce en una morfología de la cual no puede prescindir (Lefebvre, 1978, p. 67). Para ello, en la medida en que interesa aludir a una cualidad de las 
concentraciones urbanas, utilizaremos el concepto de 'centralidad', entendida como la "capacidad de un lugar de ser centro, reconocido, utilizado o apropiado socialmente como un espacio de confluencia" (Mayorga \& Fontana, 2012, p. 15). De esta manera, el concepto de centralidad permite aproximarnos al centro urbano como espacio social, en la perspectiva de Lefebvre (2013), es decir, englobando la triada conceptual de la práctica espacial, las representaciones del espacio y los espacios de representación (p. 92). En otras palabras, se prestará atención a las prácticas espaciales en tanto maneras en que se despliegan los usos y las representaciones del lugar, a través de la percepción ciudadana.

Partiendo de una mirada estrictamente urbanística, Panerai y Mangin (2002) proponen diferenciar tres categorías de centros: los centros históricos, los centroscorredor y los grandes centros comerciales (p. 248). En el primer caso, la concentración es fruto de la superposición de actividades comerciales e institucionales, donde además existe una concentración simbólica del poder. En el segundo caso, el elemento central es la accesibilidad, al situarse el centro-corredor en una vía arterial de la ciudad, ofreciendo concentración de ofertas para el consumo, la recreación y el ocio. Finalmente, afirman los autores citados que los grandes centros comerciales se erigen como nuevos núcleos al acaparar una enorme demanda de diversidad de clientes sobre la base de nuevas modalidades de consumo.

Si bien esta propuesta ayuda a entender tipologías de centros en el tejido urbano, aún es insuficiente como aproximación al espacio social que configuran. En esta perspectiva, resulta importante considerar las características del espacio público en el estudio de las centralidades urbanas. Para ello nos apoyamos en la tesis de Gehl (2006, p. 17), en el sentido de que un espacio público con buen diseño urbano se convierte en un factor estimulante para la generación de actividades opcionales, que son aquellas en que existe el deseo de realizar alguna actividad no prevista si el tiempo y lugar lo permiten. Asimismo, consideramos pertinente el enfoque de "urbanismo del espacio público" como base de la construcción de centralidades urbanas que propone Borja (2013, pp. 39-40).

Actualmente existen diversas modalidades de centros urbanos, y también de formas en que se consolidan como tales. Una tendencia mundial es el desarrollo de modelos policéntricos, donde los centros pueden localizarse indistintamente en zonas céntricas o periféricas (Capel, 2003), fenómeno definido como policentrismo descentralizado si ocurre como resultado de la descentralización del empleo, o policentrismo christalleriano si ocurre por la integración funcional de centros de menor tamaño (Muñiz, Sánchez \& García López, 2015, p. 77).

La globalización de la economía ha estimulado la concentración de servicios financieros y comerciales en diferentes lugares de la estructura urbana, que han ido ganando visibilidad internacional como puntos estratégicos para localizar sedes empresariales o sucursales de grandes empresas transnacionales. Esta concentración de actividades ha permitido que esos centros de la ciudad se erijan como nodos articulados a dinámicas económicas de carácter global. La noción de 'nodo' está asociada a una forma de ocupación del territorio caracterizada por su gran conectividad, pero a la vez por su pobre inserción en el tejido urbano. Los nodos se conciben, entonces, como centros globalmente visibles en las redes del espacio de 
los flujos, pero invisibles como lugares, es decir, como elementos de la ciudad en que se insertan (Castells, 1997). Por otra parte, las redes nodales a que aquí se alude se insertan en contextos económicos donde el llamado 'nuevo espacio industrial' ha dejado atrás las zonas fabriles como íconos de la economía, para concentrar su atención en los centros de decisión e innovación empresarial (Borja \& Castells, 2000, pp. 49-50).

En términos del territorio en que se insertan los nodos en referencia, el capital inmobiliario ha sabido observar el enorme potencial de enriquecimiento que ofrecía un suelo urbano cuyos valores, irrisorios en el mercado mundial, generaban una rentabilidad importante a través de la inversión en productos inmobiliarios, los mismos que están transformado el paisaje urbano de América Latina desde inicios del siglo xxi. Pero, a la vez, dichos productos tienden a homogeneizar las ciudades, en la medida en que son arquitectónicamente similares unos a otros en la escala global (De Mattos, 2008, p. 29), proceso observado a escala mundial por Castells (1997), quien sostiene que la llegada del espacio de los flujos opaca la relación significativa entre arquitectura y sociedad (p. 452).

La inversión privada desempeña un rol importante en la transformación de las ciudades latinoamericanas, que tradicionalmente concentraban sus actividades en una gran área central, la cual se diferenciaba de una periferia conformada principalmente por barrios marginales, estructura morfológica que tradicionalmente se ha identificado como un modelo centro-periferia. Este modelo se halla en plena transformación por la emergencia de nuevas concentraciones, pero a menor escala, en lo que es definido como la ciudad fragmentada (Bahr \& Borsdorf, 2005, p. 212).

Queda aún el interrogante referido a la cualidad urbana de los nodos, pues - como seńala Castells-, ellos tienden a independizarse de los lugares en los cuales se localizan, lo que ha llevado a Sassen (2007) a plantear la necesidad de recuperar la categoría de lugar y de proceso de producción para el análisis de la economía global (p. 126). Borja (2003), por su parte, propone aproximarse a estas configuraciones a partir de los espacios públicos, en los cuales se realizaría la síntesis de lugares y flujos (p. 119); esto es, entre el espacio urbano que ocupan estos nodos y las redes globales facilitadas por las tecnologías de la información y la comunicación, en la denominada "era de la información" en que se insertan.

En América Latina, el estudio de centralidades ha sido llevado a cabo por numerosos investigadores, principalmente en los campos de la economía y la geografía urbana, que han observado configuraciones de la densidad de empleo (Muñiz et al., 2015) al igual que otro tipo de variables, como las de localización y composición del sector terciario (Vecslir \& Ciccolella, 2011) o de densidad comercial y precios del suelo (Truffello \& Hidalgo, 2015). También es de mencionar el estudio de los cambios ocurridos en los denominados centros de carácter histórico (Carrión, 2009). Sin dejar de reconocer la importancia de estos trabajos, aquí se prestará mayor atención a la condición del centro como lugar.

La referencia al lugar, ya aludida por trabajos como los de Borja, Castells o Sassen, en muchos casos tiene como punto de partida la experiencia cotidiana de las personas. Esta experiencia convierte el lugar en un espacio social particular cuya ocupación frecuente lo vuelve inteligible para un grupo humano determinado, que 
a su vez puede incorporarlo como dispositivo espacial que exprese su identidad en tanto grupo (Augé, 1993, p. 51).

En ese sentido, una aproximación como la de Duhau y Giglia (2008) es sugerente en la medida en que invita al análisis concreto de una concentración urbana como experiencia metropolitana, a partir de la cual diferentes actores dan una particular vitalidad a un espacio construido. En el caso de la Ciudad de México, examinado por estos autores, se trata de un espacio urbano en el cual coexisten el comercio informal y el hipermercado fruto de la inversión privada. Igualmente es inspirador el trabajo de Beuf (2010), que examina los atributos de centralidad que observa en casos concretos en la periferia de Bogotá. Tomando en consideración estos enfoques, en la aproximación que aquí se plantea interesan las características del centro urbano como espacio social, su importancia como destino en los ritmos cotidianos de los habitantes de las ciudades, así como las representaciones que sobre ellos construyen quienes los ocupan o utilizan.

\section{La transformación de Lima en las décadas recientes y sus centros urbanos}

Como una primera aproximación a los centros urbanos de Lima, el estudio de Chion (2002) fue sugerente, pues identifica concentraciones a partir de actividades predominantes a finales del siglo $\mathrm{xx}$, como las financieras y comerciales, las industriales, y otras como las de esparcimiento. Sobre esa base destaca nodos urbanos, entre ellos el llamado distrito financiero de San Isidro o la zona comercial-industrial de Gamarra. Chion no encuentra centros de carácter metropolitano fuera del área central de la ciudad, aunque destaca la importancia de centros distritales en actividades comerciales o industriales en zonas de expansión, como Lima Norte, Lima Este o Lima Sur.

Los principales centros identificados por Chion se confirman como tales con la investigación de Gonzales y Del Pozo (2012), quienes encuentran que las grandes concentraciones del empleo se sitúan en la zona central de la metrópoli, sobre un eje donde las distancias no suelen exceder de siete kilómetros, cuando de sur a norte la ciudad puede cubrir distancias superiores a los ochenta kilómetros. Es en esta perspectiva que afirman que Lima experimenta una suerte de "policentrismo centralizado" (p. 49), según el cual la localización de las principales concentraciones de empleo indicaría la reproducción, a mayor escala, de las grandes desigualdades que existen entre la zona central de la metrópoli y las grandes zonas de expansión. Esta tendencia se vería confirmada por el incremento del valor del metro cuadrado del suelo urbano en los centros de Miraflores y San Isidro, que se ha multiplicado por diez en cinco años.

En cuanto a la localización de los principales centros de educación superior y grandes zonas comerciales, estos se encuentran en buena parte en el área central, aunque el eje vial de la carretera Panamericana cumple un papel importante como centro, en la perspectiva urbanística de Panerai y Mangin (2002). Al momento de superponer la información de las variables antes mencionadas, se obtiene una primera aproximación a los principales centros urbanos de la metrópoli (figura 1). 
FIGURA I | Ubicación de principales centros según las grandes zonas de Lima Metropolitana

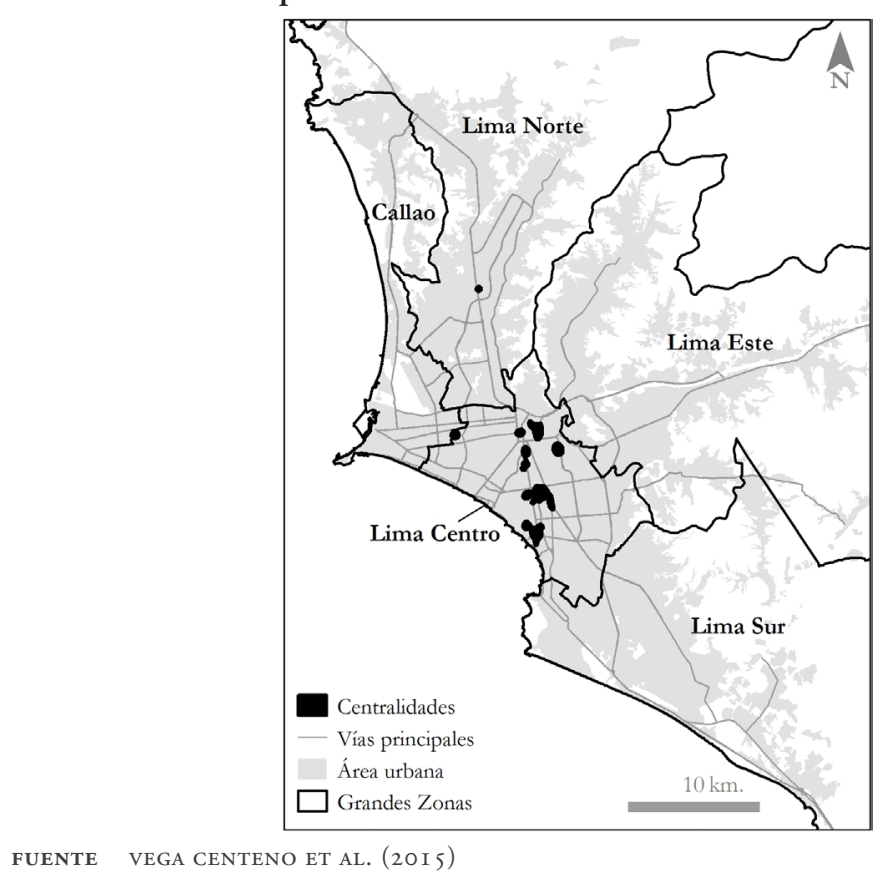

De acuerdo con la figura 1, los mayores centros metropolitanos se encuentran en su totalidad en el área central, lo que indicaría que pese a los cambios del siglo xxI, Lima mantiene aún una estructura centralizada.

No obstante, existen concentraciones en las grandes zonas de expansión cuya importancia y magnitud han aumentado, llevando a que exista movimiento de mercado inmobiliario e inversiones privadas en ellas. Esto va de la mano con la densificación que ocurre en la ciudad en términos residenciales, pues los distritos que albergaban población de origen de barriadas han aumentado notablemente su número de habitantes, sin que haya existido un incremento significativo de la superficie ocupada (Vega Centeno, 2014). Cabe entonces preguntarse si en el caso de las zonas de expansión de Lima existen centros que se expliquen primordialmente a partir del crecimiento de la densidad residencial de proximidad.

En efecto, a pesar de que los edificios de mayor altura se concentran en el área central de la ciudad, esta zona -contra lo que se puede suponer- ha dejado de ser la más densa en términos residenciales. Según indican los cambios ocurridos entre los censos de 1993 y 2007, así como las proyecciones actuales (figura 2), las áreas que concentran mayor población residencial se hallan sobre todo en Lima Norte y, luego, en Lima Este y Lima Centro. Esto cambia completamente el panorama con respecto a lo que ocurría en Lima hace veinte ańos, cuando las zonas de expansión tenían una densidad residencial muy inferior a la zona central de la metrópoli. 
FIGURA 2 | Densidad poblacional de Lima según lugar de residencia: 1993 y 2013

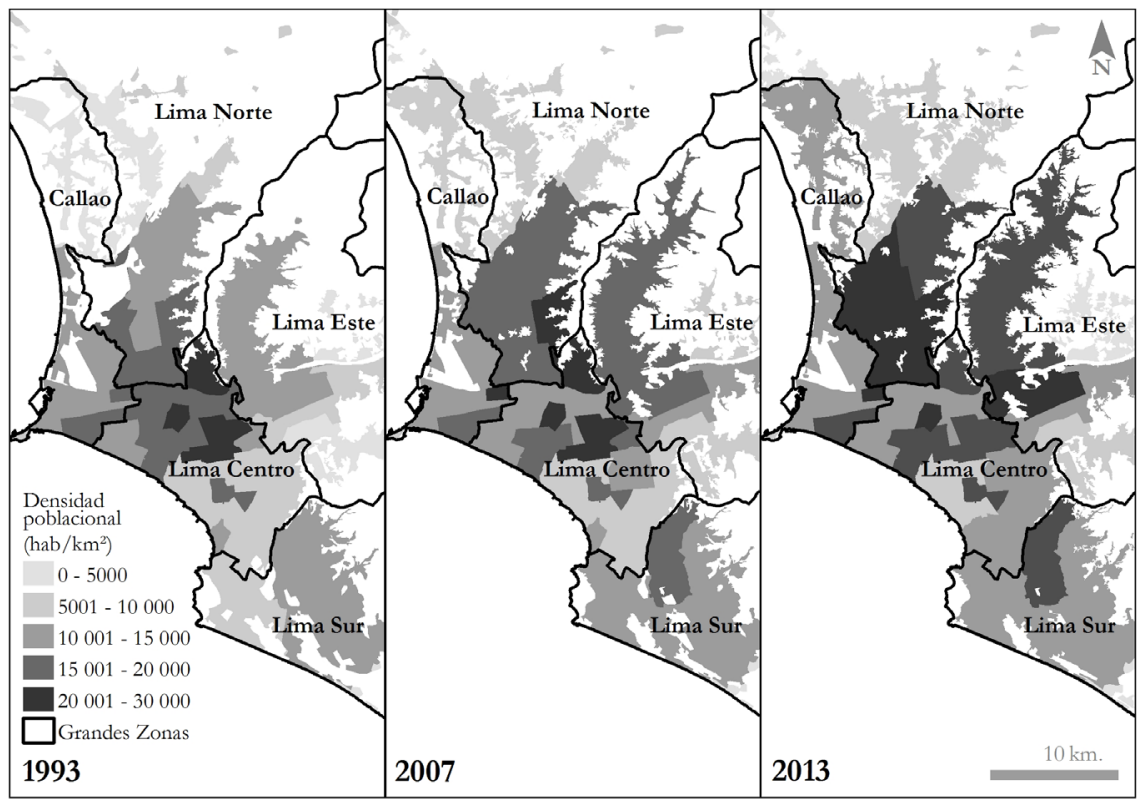

FUENTE VEGA CENTENO ET AL. (20I5)

Este nuevo panorama urbano abre interrogantes sobre dónde resuelve esta población necesidades cotidianas como el trabajo, el estudio o el abastecimiento de bienes para sus hogares. Cabe señalar que el dinamismo económico que ha venido ocurriendo en estas zonas, particularmente en Lima Norte, ha llamado la atención tanto de científicos sociales como de políticos. El Plan Regional de Desarrollo Concertado (PRDC) para Lima advierte que existe un centro urbano en Lima Norte cuya dinámica urbana es mayor que la de otras zonas de expansión de Lima, refiriéndose al que se forma en el eje de la carretera Panamericana Norte, en el límite de los distritos de Independencia y Los Olivos (PRDC, 2013, p. 189). Ensayos como los de Matos Mar (2004, p. 132) sostienen que el llamado desborde popular que experimentó Lima en la segunda mitad del siglo xx ha permitido que una población de origen migrante y originalmente carente de recursos consiga mejores niveles de vida, transformando la llamada "Lima de los Conos" en una Lima con nueva fisonomía, que atrae la inversión de grandes centros comerciales y de miles de microempresas como fruto de la fuerte inversión y del trabajo, tanto individual como colectivo, de sus pobladores, que han sabido abrirse espacio en la gran ciudad. Se trata de la tesis de la ciudad emergente, aglomeración capaz de ganar competitividad gracias al emprendimiento de sus propios habitantes, muchos de ellos laborando en microempresas productivas o de servicios a relativa proximidad de sus hogares. Según Matos Mar, Lima Norte es la zona que ha experimentado mayor movimiento de capitales, destacando grandes inversiones en complejos comerciales o hipermercados, aunque, por otra parte, reconoce que solo el $25 \%$ de la población se halla en condición de no pobre (pp. 134-135). 
Por su parte, autores como Arroyo y Romero (2009) o Joseph (2005) coinciden en destacar el dinamismo económico adquirido por esta gran área de expansión de la ciudad, Lima Norte. Joseph (2005) resalta el importante potencial empresarial de la zona hacia inicios del siglo xxi, pese a que en ella se experimentó el duro impacto de la recesión económica que redujo el área dedicada a la industria -principalmente ensamblaje de vehículos de firmas como Volvo o Volkswagen- en las últimas décadas del siglo xx; pero advierte que este dinamismo depende principalmente de micro y pequeñas empresas y unidades económicas de comercio y servicios, de las cuales solo el 24\% tendría capacidad de crecimiento (pp. 191-193). Arroyo y Romero (2009), por su parte, sitúan Lima Norte como el espacio económico más dinámico entre las grandes zonas de expansión de la ciudad (p. 127); destacan la presencia de grandes cadenas comerciales generadoras de un movimiento comercial que representaría el $41 \%$ del mercado metropolitano, lo que ayuda a prefigurar las concentraciones donde se localizan, que estos autores definen como 'centralidades emergentes'. En este contexto, sostienen que si bien el polo financiero comercial de San Isidro y Miraflores se ha fortalecido, existe una desconcentración producida por la suma de la economía local y global, que se expresa en las centralidades emergentes (p. 144).

De otro lado, documentos más orientados a los estudios de mercado, como el trabajo de Arellano y Burgos (2010), han tenido enorme impacto en los medios de opinión y los discursos políticos, lo que hace pertinente mencionarlo. Estos autores destacan el espíritu emprendedor de los habitantes de Lima Norte, que ha sabido ser atractivo para la inversión de firmas comerciales; en sus palabras, "se ha constituido en la 'punta de lanza' de una revolución comercial que sobrecoge a Lima Conurbana y que parece proyectarse a los otros lugares de la ciudad” (p. 193). Asimismo, señalan, otro campo que viene siendo importante fuente de inversión es el de los estudios superiores, que acoge el "gran deseo de superación de las personas de Lima Conurbana” (p. 193). De acuerdo con su análisis, ese afán de superación ha llevado a que negocios modernos se decidan por invertir en esta zona, lo que a su vez permitirá que su población desarrolle una mayor exigencia respecto a la calidad y variedad de servicios, convirtiéndola en un ícono de potencial desarrollo. Es más, los autores afirman que paulatinamente las zonas de expansión de la ciudad funcionarán de manera más autónoma e independiente de la zona central, pues, según ellos, el "neolimeño que hoy vive en Lima Conurbana cada vez más trabaja en su zona, y mientras esta misma zona encuentra más bienes y servicios, menos va a necesitar trasladarse a Lima Central” (p. 196).

Tanto los estudios de ciencias sociales como los de mercado abren un interrogante acerca de la real magnitud del cambio a nivel de centralidades que viene ocurriendo con la densificación y consolidación de las áreas de expansión de la ciudad, que en el siglo xx fueran sinónimo de periferias compuestas casi en su totalidad por barriadas o urbanizaciones populares. ¿Existen realmente centros emergentes capaces de constituirse en centralidades de escala metropolitana? En esta perspectiva, es necesario observar con mayor detenimiento las características de estos centros urbanos identificados en Lima, prestando especial atención a indicadores de movilidad y de representación. Al respecto, consideramos que estos llamados centros urbanos de Lima Norte satisfacen principalmente necesidades cotidianas de su entorno residencial, pero sin necesariamente alcanzar una dimensión de centralidad metropolitana. 


\section{Metodología}

En el presente trabajo se presentan los primeros resultados obtenidos por la investigación del Centro de Investigación de la Arquitectura y la Ciudad (CIAC) sobre la densificación urbana de Lima en el siglo xxi (Vega Centeno et al., 2015), mediante observación de campo, encuestas y sondeos de opinión. ${ }^{1}$ Con la información recogida se espera lograr dos objetivos. Por una parte, identificar la importancia de las concentraciones ubicadas en Lima Norte con relación al conjunto de centros que estructuran la metrópoli. Por otra, detectar las percepciones que sobre los centros ubicados en Lima Norte tienen sus propios usuarios, para lo que se realizará un análisis comparativo con el caso de la centralidad comercial de Miraflores, en el área central de Lima. Se eligió este último distrito como referencia, en la medida en que es reconocido por numerosos estudios como uno de los más importantes centros urbanos de escala metropolitana de la ciudad.

Para la identificación de los principales centros urbanos de una ciudad, se suele partir del grado de concentración del empleo, de la conectividad o de la densidad de establecimientos comerciales. En este caso, interesa identificar la forma en que estas concentraciones satisfacen necesidades cotidianas de los habitantes de Lima; para ello, se tomará como primeros indicadores los viajes cotidianos de la población limeña según la gran zona en que habita, así como los del lugar de residencia y motivo de viaje de los usuarios de determinadas concentraciones; también se incluyen referencias a los modos de desplazamiento, en la medida en que permiten identificar algunas de las semejanzas y diferencias del área central de Lima, o Lima Centro, con relación a sus zonas de expansión. Por otra parte, se busca una primera aproximación a las percepciones de los usuarios de los centros urbanos a los que acuden en tanto lugar con el que pueden establecer referencias identitarias, para lo cual se toman como indicadores las percepciones de seguridad, limpieza, calidad del tráfico urbano y de las veredas para peatones.

\section{Identificando la importancia de los centros de Lima Norte}

La encuesta de movilidad aplicada muestra que se mantiene la importancia del área central de la ciudad para el conjunto de la urbe, pues la población que reside en esta macrozona es la que menos viajes interzonales realiza en su vida cotidiana. No obstante, existen matices importantes de considerar. El principal motivo de desplazamiento está relacionado con aspectos laborales, seguido por el de compras, tendencia presente en las distintas zonas de la urbe, como se observa en la tabla 1. Resulta pertinente, entonces, observar con algo más de detenimiento lo que ocurre con los viajes por trabajo o por compras.

1 Como parte del material empírico producido en la investigación, se aplicó una encuesta de movilidad a una muestra de 1200 casos, con 95\% de nivel de confianza, para identificar destinos laborales, educativos y de abastecimiento de los habitantes de Lima Metropolitana. Asimismo, se aplicaron sondeos a muestras de aproximadamente 400 casos para dos concentraciones de Lima Norte, así como para concentraciones de reconocida importancia metropolitana, como la zona comercial de Miraflores. 
TAbla I | Motivo del desplazamiento principal de los habitantes de LimaCallao según gran zona en la que reside (en porcentajes)

\begin{tabular}{|l|c|c|c|c|c|}
\hline $\begin{array}{c}\text { MOTIVO DEL } \\
\text { VIAJE }\end{array}$ & LIMA CENTRO & LIMA NORTE & LIMA ESTE & LIMA SUR & CALLAO \\
\hline Trabajo & 45 & 42 & 47 & 48 & 35 \\
\hline Estudios & 9 & 9 & 9 & 8 & 8 \\
\hline Compras & 24 & 24 & 25 & 22 & 25 \\
\hline Ocio & 6 & 6 & 2 & 5 & 7 \\
\hline Otros & 16 & 16 & 17 & 16 & 21 \\
\hline
\end{tabular}

FUENTE ELABORACIÓN PROPIA A PARTIR DE ENCUESTA APLICADA EN EL MARCO DEL ESTUDIO DEL CIAC-PUCP (2OI4) SOBRE LA DENSIFICACIÓN URBANA DE LIMA EN EL SIGLO XXI (VEGA CENTENO ET AL., 2OI 5)

Con respecto a los viajes que se realizan por motivos laborales, el $72 \%$ de los que residen en Lima Centro viajan a un destino localizado en la misma zona (figura 3). En cambio, cerca del 50\% de quienes residen en otras áreas de la ciudad tienen que hacer extensos viajes interzonales, siendo el caso más llamativo el de Lima Sur; hay pues algo más de 20 puntos porcentuales de diferencia entre la capacidad de acoger viajes laborales de sus propios residentes en el Centro con relación a lo que ocurre en las otras grandes zonas de la metrópoli. ${ }^{2}$ De esta forma, pese a la dinámica económica generada en centros localizados en Lima Norte, estos no demuestran capacidad de absorber sino una parte del conjunto de población económicamente activa que ahí reside, situación que afecta la organización del transporte en la ciudad, si se tiene en cuenta que ahí se localizan los espacios residenciales más densos de la metrópoli, como se ilustró en la figura 1.

FIGURA 3 | Viajes por motivos laborales dentro de la gran zona según zona de residencia

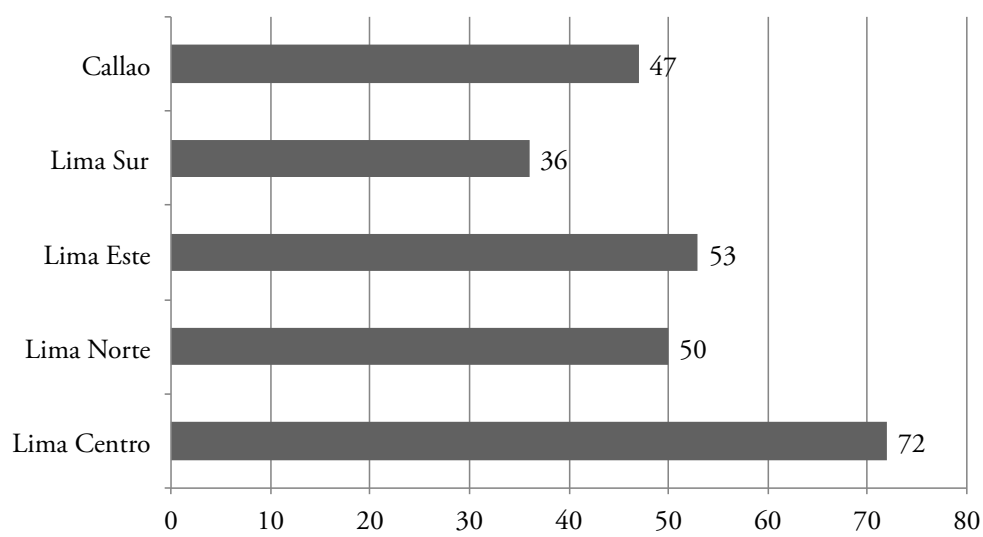

FUENTE ELABORACIÓN PROPIA A PARTIR DE ENCUESTA CIAC-PUCP, JULIO 20 I4 (VEGA CENTENO ET AL., 2OI 5)

2 Un trayecto interzonal supone habitualmente un viaje metropolitano que supera los veinte kilómetros de distancia. 
Un efecto de lo anterior será la necesidad de medios motorizados para resolver largos viajes al trabajo. Como se observa en la figura 4, el principal modo de desplazamiento utilizado en cualquiera de las grandes zonas de Lima es el transporte público, que representa largamente más de la mitad de las opciones, aunque siempre más significativo en las áreas de expansión.

\begin{tabular}{l|l} 
FIgURA 4 & $\begin{array}{l}\text { Modo de desplazamiento por motivos laborales según zona de } \\
\text { residencia }\end{array}$
\end{tabular}

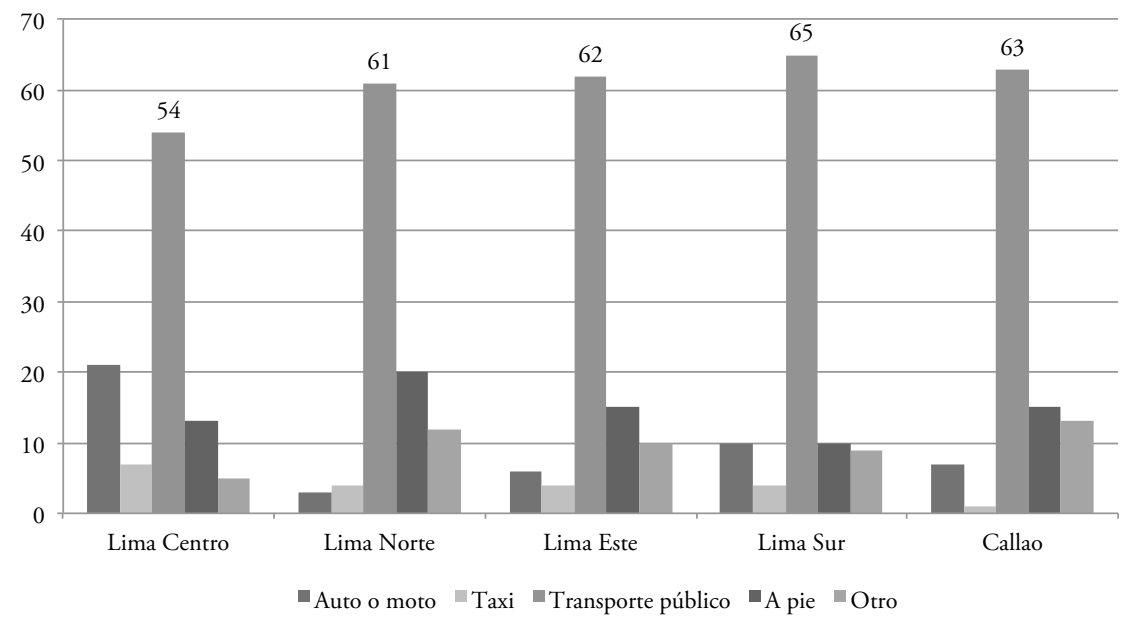

FUENTE ELABORACIÓN PROPIA A PARTIR DE ENCUESTA CIAC-PUCP, JULIO 20 I 4 (VEGA CENTENO ET AL., 20I 5)

Es llamativa la concentración del uso del coche privado en la zona central de la ciudad, pese a que sus habitantes son los que menos viajes interzonales realizan. En Lima Centro, el uso de motocicletas o automóviles propios asciende al $21 \%$, mientras que en el resto de la metrópoli este modo de desplazamiento representa el $10 \%$ o menos. Los viajes peatonales por motivos laborales son minoritarios, aunque es significativo que el $20 \%$ de adultos entrevistados en Lima Norte sí acuda a pie a su lugar de trabajo. En suma, partiendo de los espacios residenciales, los habitantes del área central de la ciudad son los que tienen menor necesidad de viajar fuera de la gran zona en la que residen por motivos laborales, mientras las posibilidades de empleo concentradas en las grandes zonas de expansión de la metrópoli solo tienen capacidad de cubrir la mitad de las necesidades laborales de su propia población residente.

$\mathrm{Si}$ se toman en cuenta los viajes destinados a realizar compras, el panorama es distinto; las diferencias interzonales se reducen notablemente. Como se observa en la figura 6, salvo el caso de Lima Sur, no existen más de 10 puntos porcentuales de diferencia entre las respuestas de Lima Centro con respecto a otras zonas metropolitanas. Es significativo, además, que en promedio tres de cada cuatro viajes que se realizan por motivo de compras no busquen un destino fuera de su gran zona. Esto indicaría que la oferta de mercados, centros comerciales o tiendas en general está mucho mejor 
distribuida dentro de la ciudad, lo que reduce la necesidad de viajes interzonales para cubrir diferentes necesidades de abastecimiento del hogar y de sus integrantes.

\section{FIGURA 5 Destino de viajes por compras según zona de residencia}

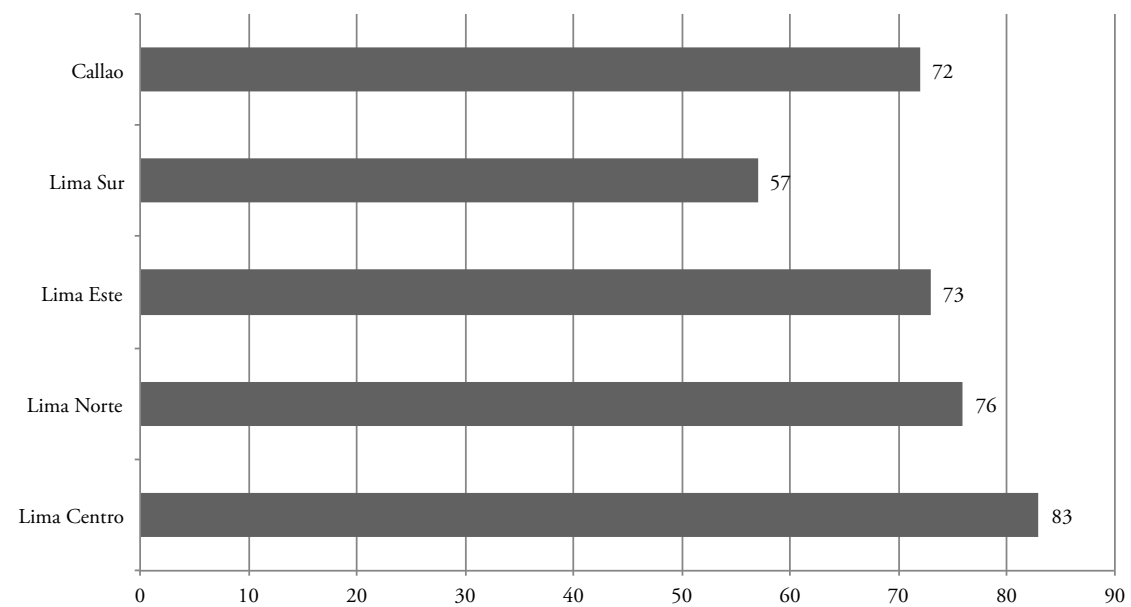

FUENTE ELABORACIÓN PROPIA A PARTIR DE ENCUESTA CIAC-PUCP, JULIO 20 I 4 (VEGA CENTENO ET AL., 2OI 5 )

FIGURA 6 | Modo de desplazamiento por motivos de compras según zona de residencia

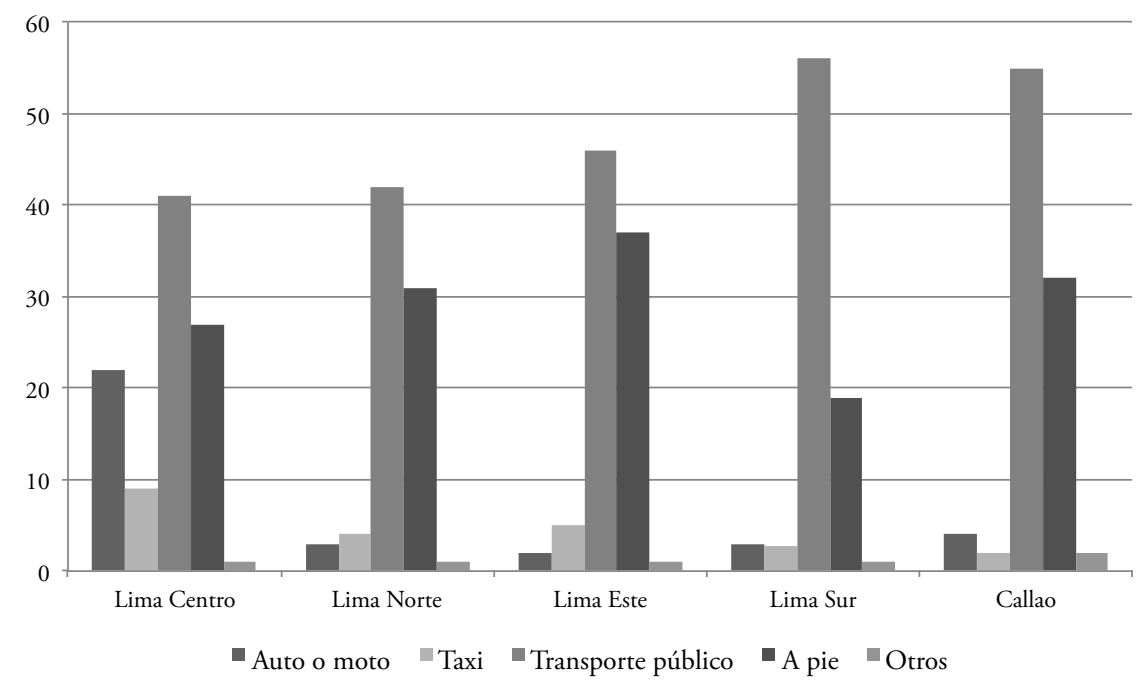

FUENTE ELABORACIÓN PROPIA A PARTIR DE ENCUESTA CIAC-PUCP, JULIO 20 I4 (VEGA CENTENO ET AL., 20I 5) 
Como consecuencia de lo anterior, es lógico que los trayectos a pie aumenten, hasta representar cerca de la tercera parte del total de viajes, aunque el transporte público es siempre el principal medio utilizado. Llama la atención, eso sí, que es nuevamente Lima Centro la única zona donde el uso del auto propio tiene alguna significancia, al cubrir el $21 \%$ de los modos de desplazamiento utilizados, lo cual se explica en parte porque en esta zona se concentran los sectores sociales de más altos ingresos económicos. En otras palabras, los viajes por motivos de compra tienden a encontrar destinos acordes a las necesidades de los usuarios en lugares no tan distantes de donde residen, lo que indicaría una descentralización de la oferta de equipamientos comerciales muy superior a lo que ocurre con los espacios laborales.

Si bien esta información no precisa el destino final de los viajes, sí permite relativizar ciertas afirmaciones que se hacen en torno a la expansión económica de las llamadas zonas emergentes de Lima, y en particular Lima Norte. En efecto, mientras cerca de la mitad de residentes necesita viajar fuera de su gran zona por motivos laborales, resulta excesivo afirmar que los habitantes de Lima Norte necesitan cada vez menos trabajar fuera de su gran zona. En cambio, es cierto que sí existe una mejor distribución de las ofertas de equipamientos comerciales, a lo cual es posible que haya aportado la descentralización de inversiones en grandes tiendas comerciales y, sobre todo, en enormes centros comerciales o malls, pero estas inversiones por sí solas no permiten afirmar la existencia de una potencial centralidad metropolitana. Para pronunciarse al respecto, es necesario observar con mayor atención la dimensión urbana de aquellas concentraciones ubicadas en zonas de expansión. A tal efecto, se examinarán dos de los principales centros detectados al momento de relacionar variables como las del empleo y accesibilidad con las de movilidad y servicios. Ambos se localizan en el eje de la carretera Panamericana Norte, uno en el límite distrital de Independencia con Los Olivos y otro en el distrito de Puente Piedra. En términos operativos, tales concentraciones serán identificadas como el Centro Comercial Independencia y Centro Comercial Puente Piedra, atendiendo a que es esta rama de actividad la que otorga el mayor dinamismo económico a ambos lugares.

\section{Características de los centros de Lima Norte en comparación con la centralidad comercial de Miraflores}

La principal concentración de actividades en Lima Norte es la centralidad comercial Independencia, donde hasta la década de 1980 operaba uno de los más importantes parques industriales de la ciudad y que hoy constituye uno de los principales destinos de viajes cotidianos de la zona. Con la gran recesión económica de aquella década, que supuso el fin de la política de industrialización sustitutiva de importaciones, grandes industrias cerraron, quedando lotes enormes en desuso. Fue en tal contexto que importantes grupos inversores decidieron ocupar estos terrenos para habilitar en ellos centros comerciales de enorme envergadura. El inicio de operaciones de estos shopping centers tuvo un enorme impacto, pues era la primera vez que los malls, con su formato de uniformidad global, se localizaban fuera del área central de la metrópoli. Para la simbología de muchos, ello suponía una suerte de reconocimiento de que en las áreas de expansión de Lima también se generaban atractivos económicos que ameritaban una suerte de visibilidad nodal. 
FIGURA 7 Delimitación de centros de Lima Norte y usos que concentran

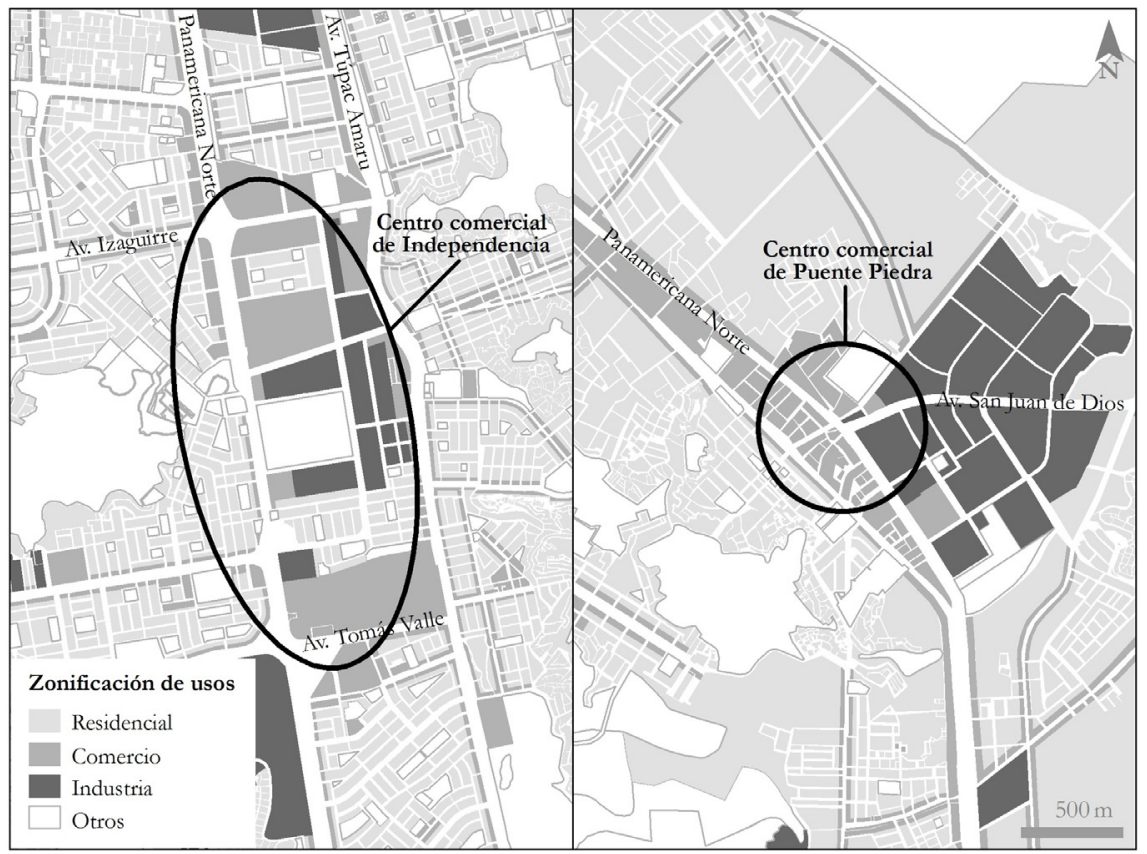

FUENTE VEGA CENTENO ET AL., 2015

En la actualidad, esta centralidad concentra, además de actividades comerciales, zonas residenciales y otros usos, como espacios educativos o recreacionales (figura 7). La concentración también incluye predios de uso industrial, donde la actividad predominante es la metalmecánica. De acuerdo con el sondeo aplicado, cerca del $80 \%$ de los usuarios que acuden a esta centralidad provienen de distritos que forman parte de la misma gran zona. Entre ellos, el $28 \%$ acude a esa concentración por motivos laborales, $16,5 \%$ para realizar algún tipo de trámite, $14,5 \%$ a pasear y un $10,5 \%$ por compras. Asimismo, un significativo 16,5\% utiliza esta concentración como conexión de transporte para un destino más alejado. Por último, también existe un importante $16,5 \%$ que acude a este lugar por razones de estudio. Según entrevistas realizadas en la zona, varios comerciantes, tanto con locales fijos como ambulantes, señalan que la implementación del corredor segregado de buses "El Metropolitano" y su estación final situada unas cuadras más al norte de donde se ubican los centros comerciales ha multiplicado la afluencia de transeúntes, lo cual se convierte en oportunidades de negocio para diferentes actividades de comercio y servicios.

Una segunda concentración significativa es la que se ha venido generando también sobre el eje de la carretera Panamericana Norte, pero esta vez en el distrito de Puente Piedra. Se trata de la conurbación de un antiguo pueblo republicano, reconocido como distrito desde 1927 y que fue lugar de recreación campestre para los limeños hasta la década de 1980. Fue recién hacia fines de aquella década que terminó por conurbarse con el área metropolitana de Lima, convirtiéndose en una 
importante zona de expansión para las urbanizaciones más recientes. En las proximidades del núcleo central del antiguo pueblo, sobre el eje carretero, coexisten áreas destinadas a la actividad industrial y al uso residencial, las cuales paulatinamente han cedido en importancia frente a la intensa actividad comercial que ha venido dominando esta concentración las últimas décadas (figura 7).

En este centro sobresale la gran aglomeración de puestos comerciales reunidos en el mercado Huamantanga, que cuenta con cerca de mil asociados, además de otras asociaciones de comerciantes que ocupan espacios aledańos y encadenan en su entorno galerías comerciales y tiendas de mediana y gran escala. Asimismo, existen servicios financieros ubicados sobre el eje vial de la carretera, y a poco más de 300 metros de este eje vial se encuentran la municipalidad, la iglesia matriz y algunas instituciones educativas, mientras a pocos metros del mercado se ubica una importante zona destinada a la actividad industrial, predominando el ramo de industrias alimentarias o de bienes semidurables. En este caso, el $87 \%$ de la población sondeada reside en la zona norte de Lima, destacando que la mayor parte $(56 \%)$ habita en el propio distrito. De este universo, el 29\% acude a esta concentración por motivos laborales y otro tanto para realizar compras (29,5\%). También es importante el número de personas que solo están de paso (19,8\%), es decir, que acuden a esta concentración como escala que los conecta hacia destinos más alejados de su espacio residencial.

En suma, nos encontramos ante dos centros que tienen como característica común el contar con varias manzanas destinadas al uso industrial, pero donde la actividad predominante es el comercio. La diferencia entre ambos es que mientras en Puente Piedra los principales actores son las asociaciones de numerosos pequeños comerciantes, en Independencia los grandes centros comerciales ahí localizados tienen un rol significativo.

Ahora bien, ¿qué puede decirse de sus cualidades urbanas? En ambos casos hay buena accesibilidad, esto es, para cerca del $70 \%$ de entrevistados es fácil o muy fácil llegar al lugar. Sin embargo, las percepciones del tránsito son muy negativas en ambos casos. Lo mismo ocurre con la percepción de la calidad de las veredas, la limpieza y la seguridad en general. Y si bien el distrito de Independencia tiene mayor nivel de consolidación que Puente Piedra, condición que se expresa en un mayor crecimiento vertical de las edificaciones, en ambos llama la atención la pobre calidad de diseño tanto de los espacios destinados a la circulación vehicular como de aquellos por donde transitan los peatones. El flujo peatonal es importante, y aun así debe discurrir entre muchos ambulantes que ocupan espacios de aceras, para luego inventar trayectos que permitan atravesar de manera audaz intersecciones sin señalización ni semáforos que ofrezcan alguna protección o prioridad al caminante. Esto es manifestado por los entrevistados en ambas localidades, donde los peatones se ven forzados a improvisar tácticas colectivas para cruzar avenidas donde la gestión urbana no les ha prestado atención alguna.

Se identifica, pues, concentración de diversas actividades, dinámicas y flujos importantes de población, pero a la vez pobreza de la infraestructura vial y del equipamiento de los espacios públicos que arman el tejido urbano en estos lugares. Estas observaciones resultan más evidentes si se compara las percepciones registradas en 
estas concentraciones con aquellas generadas en una reconocida centralidad limeña, la zona comercial de Miraflores.

En primer lugar, al observar la procedencia de los entrevistados, se hace evidente que, en el caso de Miraflores, el poder de convocatoria de la centralidad trasciende Lima centro, pues cerca del $50 \%$ de sus usuarios proviene de otras zonas de la ciudad, inclusive algunos de otras regiones del país o del extranjero. Los centros de Lima Norte son importantes dentro de su gran zona, pero no lo son para el conjunto de la ciudad.

FIgURA 8 | Lugar de residencia de los usuarios de los centros urbanos de Independencia, Puente Piedra y Miraflores

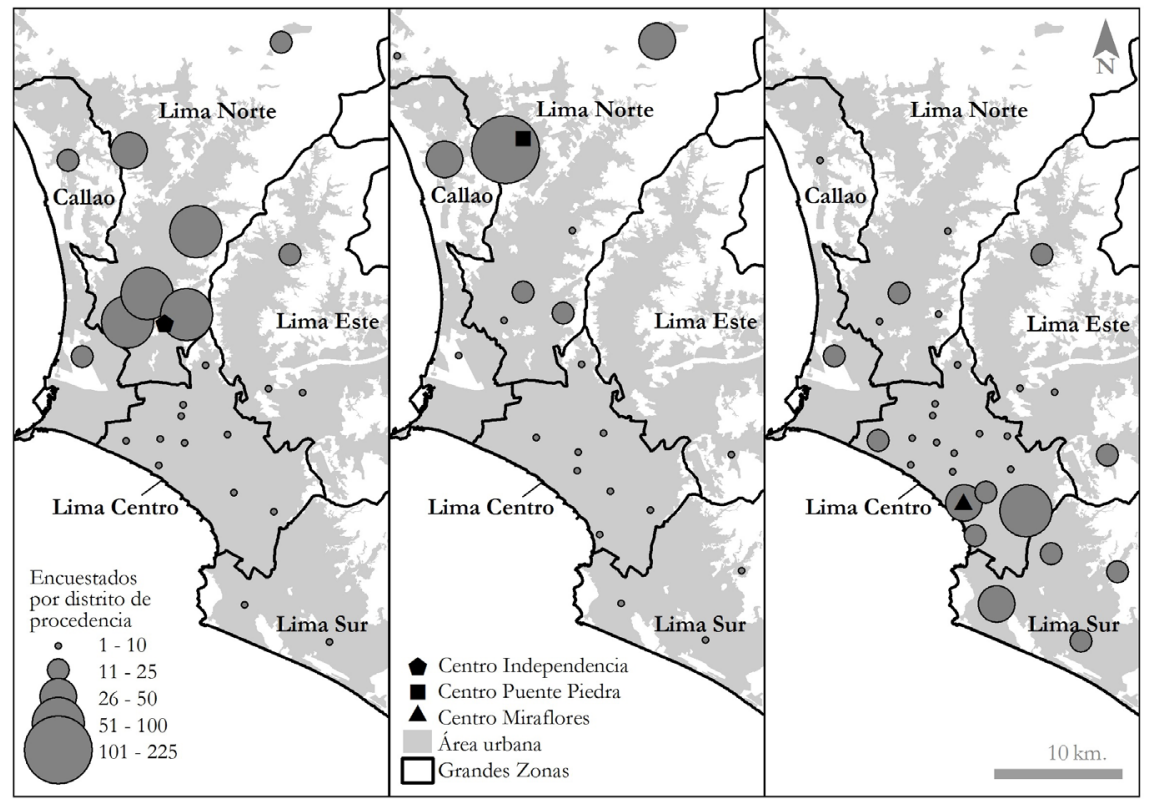

FUENTE INVESTIGACión CIAC-PUCP (VEGA CENTENO ET AL., 20I5), A PARTiR DE RESUlTAdos DE SONDEOS APLICADOS EL AÑO 20 I 4

En segundo lugar, si bien a los tres centros cerca de la tercera parte de la población acude por motivos laborales (figura 8), llama la atención que en Miraflores muchos acudan por el placer de pasear, lo que indica un poder de atracción que trasciende la necesidad de viaje obligatorio, como puede ser la generada por el trabajo o el estudio; en este caso se está ante la posibilidad de realizar actividades opcionales, en la perspectiva de Gehl (2006). Por otra parte, los centros ubicados en el norte de la ciudad reúnen más del doble de población que se encuentra de paso con respecto a Miraflores; esto indicaría que estos centros no operan solamente como destino final, sino que parte de su fuerza centrípeta se explica por ser nodo de conexión importante para viajes urbanos mayores. 
TABLA 2 Principal actividad realizada en la zona (en porcentajes)

\begin{tabular}{|l|c|c|c|}
\hline \multicolumn{1}{|c|}{ ACTIVIDADES } & CENTRO MIRAFLORES & $\begin{array}{c}\text { CENTRO } \\
\text { INDEPENDENCIA }\end{array}$ & $\begin{array}{c}\text { CENTRO PUENTE } \\
\text { PIEDRA }\end{array}$ \\
\hline Compras & 11 & 10,5 & 29,5 \\
\hline Pasear & 23 & 14,5 & 3,8 \\
\hline Comer & 3,3 & 0,2 & 0,3 \\
\hline Trabajo & 33,5 & 28,4 & 29 \\
\hline Estudios & 7,8 & 16,5 & 4 \\
\hline Trámites & 2,3 & 6,7 & 1,3 \\
\hline De paso & 7,8 & 16,5 & 19,8 \\
\hline Reside cerca & 4 & 1,7 & 11,5 \\
\hline Otros & 7,5 & 5 & 1 \\
\hline
\end{tabular}

FUENTE ELABORACIÓN PROPIA A PARTIR DE SONDEO APLICADO EN AGOSTO 20 I 4 (VEGA CENTENO ET AL., 2OI 5 )

Asimismo, cerca de una tercera parte de la población que utiliza el centro comercial de Puente Piedra acude a ese destino por motivo de compras. Esto puede vincularse a lo observado en el gráfico anterior, donde se muestra que la mayor parte de personas proviene del entorno próximo o del mismo distrito. Se trata de un centro particular que cumple como fuerza centrípeta para la población que reside en los distritos que se encuentran más al norte de Independencia y que merece un estudio a mayor profundidad sobre sus características como espacio social.

En tercer lugar, existe una notable diferencia perceptiva en torno a la calidad del espacio público en que se localizan las concentraciones, lo que puede observarse a través de cuatro indicadores: la calidad de las veredas, la limpieza de las calles, el tránsito y la seguridad (figura 9). Mientras en Miraflores el 67\% califica la calidad de las veredas como buena o muy buena, el $47 \%$ de Independencia y el $45 \%$ de Puente Piedra califican estos espacios como malos o muy malos. Algo similar ocurre con la limpieza de la zona, que el $81 \%$ califica como muy buena o buena a Miraflores, mientras que el 54\% de Independencia y el 53\% de Puente Piedra la encuentran mala o muy mala. Por otra parte, también existen diferencias significativas en la percepción del tránsito, aunque esta tampoco es muy positiva en el caso de Miraflores. En esta zona céntrica de Lima el tránsito es calificado como regular por el $41 \%$, mientras es percibido como malo o muy malo en Independencia por $71 \%$, y por $87 \%$ en Puente Piedra.

De igual tendencia es la percepción de la seguridad, donde el $78 \%$ la califica de buena o muy buena en Miraflores, cuando el $61 \%$ en Independencia y el $47 \%$ en Puente Piedra la perciben mala o muy mala. El centro urbano localizado en Miraflores es un imán de escala metropolitana cuyo espacio público es favorablemente percibido en términos generales, situación que actualmente no existe en las otras dos concentraciones, donde es poco usual encontrar a residentes de otras grandes zonas de la metrópoli y donde las percepciones de las veredas, el tránsito, la limpieza y la seguridad son de muy bajos niveles. 
FIGURA 9 | Percepción de calidad del espacio público: veredas, tránsito, limpieza y seguridad según centros

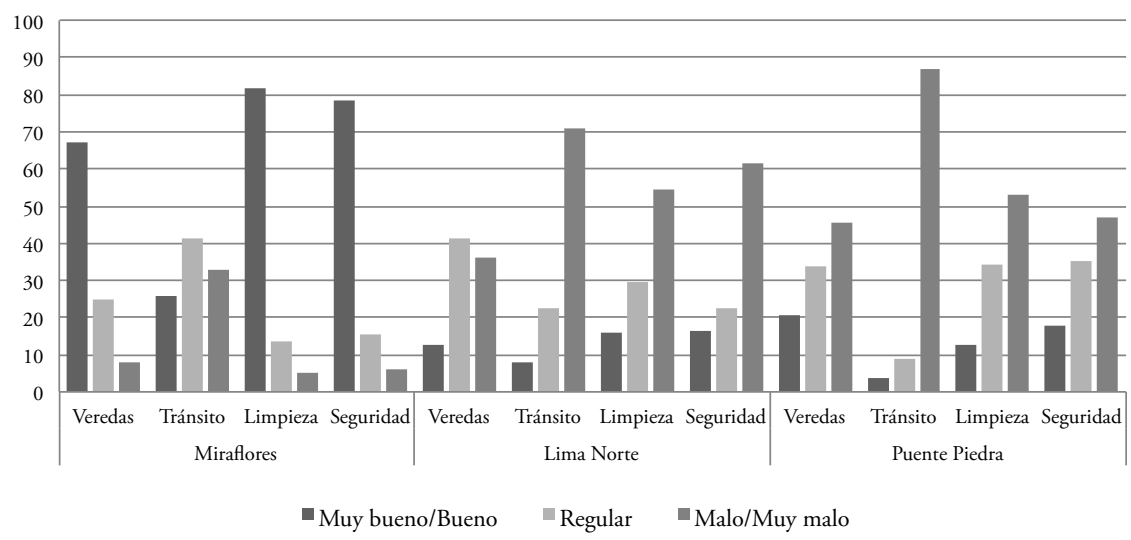

FUENTE ELABORACIÓN PROPIA A PARTIR DE SONDEO APLICADO EN AGOSTO 20 I4 (VEGA CENTENO ET AL., 2OI 5)

El enorme flujo de personas que utilizan ambas concentraciones de Independencia no va de la mano con la calidad física del espacio que ocupan. Queda además abierta la interrogante sobre cómo contribuir a una mejor percepción ciudadana en estos casos, donde es clamorosa la ausencia o insuficiencia de inversión pública en la consolidación de los espacios públicos. No se discute la importancia del notable flujo de personas que pasa por los centros de Lima Norte, pero sí es llamativa la escasa inversión pública en infraestructura y equipamiento de estas centralidades urbanas que concentran importante actividad económica.

\section{Conclusiones}

\section{Lima emergente: ilusiones y realidades}

La expansión de Lima en las últimas décadas ha ido de la mano con el desarrollo de nuevos centros urbanos, situación inevitable si tenemos en cuenta que estamos ante una metrópoli de cerca de diez millones de habitantes que ocupa un territorio sumamente extenso. En este contexto, indicadores del movimiento económico en la zona norte de Lima, como la inversión de capital privado a través de los grandes grupos que habilitan centros comerciales o por la gran concentración de comerciantes de pequeña escala, han llevado a algunos autores a afirmar que esta gran área de expansión de la ciudad viene consolidando sus propias grandes centralidades, erigiéndose como una suerte de nueva Lima, pujante y emergente.

Estudios como los de Gonzales y Del Pozo (2012) ya advirtieron que, utilizando como indicador la densidad del empleo, la estructura urbana de Lima mantenía las principales grandes concentraciones dentro de la zona central de la metrópoli. Los resultados de la encuesta de movilidad que aplicáramos encuentran una tendencia similar, cuando resulta que salvo los limeños de Lima Centro, por lo menos la mitad de los adultos que habitan en áreas de expansión necesitan viajar por motivos 
laborales fuera de su macrozona de residencia. No obstante, esta tendencia debe matizarse cuando se considera los viajes motivados por actividades de consumo o abastecimiento, en cuyo caso solo una minoría de habitantes realiza compras fuera de la gran zona donde reside.

En este escenario, es importante destacar que los espacios residenciales más densos ya no se encontrarían en el área central. Entonces, la aparición de centros de jerarquía media en zonas de expansión de la metrópoli se explicaría en una lógica de policentrismo christalleriano, donde la población presiona por contar con centros de abasto a relativa proximidad de sus hogares, tendencia que se confirma con los resultados obtenidos por los sondeos de opinión.

Por otro lado, si bien los centros de Lima Norte concentran enormes flujos de población, las calidades del espacio público son deficientes, lo cual se expresa en una percepción altamente negativa por parte de la población usuaria respecto no solo de la calidad del espacio, sino también de su seguridad. En estas circunstancias, si bien estas concentraciones cumplen un rol de centralidad para diferentes necesidades de la población residente de la propia gran zona, presentan serias deficiencias como espacios urbanos, lo que afecta negativamente la percepción que de ello tienen los usuarios.

En suma, los centros urbanos de Lima Norte, si bien han adquirido importancia para sus entornos residenciales, principalmente para fines de abastecimiento, no demuestran trascender una escala zonal en la metrópoli, pese a la presencia importante de inversión privada en centros comerciales o de numerosas microempresas. Si se aspira a desarrollar estas concentraciones como centralidades metropolitanas, será necesario prestar mayor atención a la calidad urbana de estos espacios, así como incrementar su capacidad de oferta de oportunidades laborales. Y para ello es fundamental una mayor presencia de la inversión pública, no solo en infraestructura, sino como planificación urbana que se plasme en proyectos urbanísticos que eleven la calidad del espacio público en que se insertan.

\section{Referencias bibliográficas}

Arellano, R. \& Burgos, D. (2010). Ciudad de los Reyes, de los Chávez, de los Quispe... Lima: Planeta-Arellano Marketing.

Arroyo, R. \& Romero, A. (2009). Colonizados, globalizados y excluidos en las grandes transformaciones de Lima. En M. Dammert (Coord.), Perú: la construcción sociocultural del espacio territorial y sus centralidades (pp. 107-149). Quito: Organización Latinoamericana y del Caribe de Centros Históricos (Olacchi). http://www.flacsoandes. edu.ec/web/imagesFTP/1259644496.Colonizados_globalizados__excluidos.pdf

Augé, M. (1993). Los no lugares. Espacios del anonimato. Barcelona: Gedisa.

Bähr, J. \& Bordsdorf, A. (2005). La ciudad latinoamericana. La construcción de un modelo: vigencia y perspectivas. ur[b]es, Revista de ciudad, urbanismo y paisaje, 2(2), 207-221. https:/www.uibk.ac.at/geographie/personal/borsdorf/pdfs/urbes-2-2005-207-221.pdf

Beuf, A. (2010). Nuevas centralidades y acceso a la ciudad en las periferias bogotanas. Bulletin de l'Institut Francais d'Études Andines, 4O(1), 147-178. https://bifea.revues.org/1663 
Borja, J. (2003). La ciudad conquistada. Madrid: Alianza. Versión digitalizada en https:// derechoalaciudadflacso.files.wordpress.com/2014/01/jordi-borja-la-ciudadconquistada.pdf

Borja, J. \& Castells, M. (2000). Local y global. La gestión de las ciudades en la era de la información (5 $5^{\mathrm{a}}$ ed.). Madrid: Taurus.

Borja, J. (2013). Revolución urbana y derechos ciudadanos. Madrid: Alianza.

Capel, H. (2003). Redes, chabolas y rascacielos: las transformaciones físicas y la planificación en las áreas metropolitanas. Mediterráneo económivo, (3) 199-238. http://www.publicacionescajamar.es/pdf/publicaciones-periodicas/mediterraneoeconomico/3/3-27.pdf

Carrión, F. (2009). El centro histórico como objeto de deseo. En: Varios, Seminario Permanente "Centro Histórico de la Ciudad de México" (vol. 1, pp. 17-34). México: Universidad Nacional Autónoma de México (UNAM). https://works.bepress.com/fernando_carrion/532/ Castells, M. (1997). La sociedad red. Vol. La era de la información. Madrid: Alianza.

Chion, M. (2002). Dimensión metropolitana de la globalización. Lima a fines del siglo xx. EURE, 28(85), 71-87. https://doi.org/10.4067/S0250-71612002008500005

De Mattos, C. (2008). Globalización, negocios inmobiliarios y mercantilización del desarrollo urbano. En P. C. Pereira \& R. Hidalgo (Eds.), Producción inmobiliaria y reestructuración metropolitana en América Latina (pp. 23-40). Santiago: Pontificia Universidad Católica de Chile / Universidad de São Paulo.

Duhau, E. \& Giglia, A. (2008). Las reglas del desorden. México: Siglo xxI.

Gehl, J. (2006). La humanización del espacio urbano. Barcelona: Reverté.

Gonzales, E. \& Del Pozo, J. M. (2012). Lima, una ciudad policéntrica. Un análisis a partir de la localización del empleo. Investigaciones Regionales, (23), 29-52. http://www.aecr.org/ images/ImatgesArticles/2012/10/Gonzales.pdf

Joseph, J. (2005). La ciudad, la crisis y las salidas. Lima: Universidad Nacional Mayor de San Marcos.

Lefebvre, H. (1978). El derecho a la ciudad (4a ed.). Barcelona: Península.

Lefebvre, H. (2013). La producción del espacio urbano. Madrid: Capitán Swing.

Mayorga, M. \& Fontana, M. P. (2012). Espacios de centralidad urbana y redes de infraestructura. Bitácora 21(2), 11-26. http://www.revistas.unal.edu.co/index.php/bitacora/article/ view/29084/pdf_169

Matos Mar, J. (2004). Desborde popular y crisis del Estado. Veinte años después. Lima: Fondo Editorial del Congreso del Perú.

Panerai, P. \& Mangin , D. (2002). Proyectar la ciudad. Madrid: Celeste.

Plan Regional de Desarrollo Concertado de Lima 2012-2025 (PRDC). (2013) Lima: Instituto Metropolitano de Planificación. http://sinia.minam.gob.pe/documentos/planregional-desarrollo-concertado-lima-2012-2025

Polydorides, N. (1983). The concept of centrality in urban form and structure. Serie European University Studies. Berna, Alemania: Peter Lang.

Muñiz, I., Sánchez, V. \& García-López, M. (2015). Estructura espacial y densidad de población en la zмvм 1995-2010: evolución de un sistema urbano policéntrico. EURE, 41(122), 75-102. https://doi.org/10.4067/S0250-71612015000100004

Sassen, S. (2007). Una sociología de la globalización. Buenos Aires: Kats. 
Truffello, R. \& Hidalgo, R. (2015). Policentrismo en el área metropolitana de Santiago de Chile: reestructuración comercial, movilidad y tipificación de subcentros. EURE, 41(122), 49-73. https://doi.org/10.4067/S0250-71612015000100003

Vecslir, L. \& Ciccolella, P. (2011). Relocalización de las actividades terciarias y cambios en la centralidad en la Región Metropolitana de Buenos Aires. Revista de Geografía Norte Grande, (49), 63-78. https:// doi.org/10.4067/S0718-34022011000200005

Vega Centeno, P. (2014). La dénsification récente de Lima et ses défis en matière de logement et transport. Problèmes d'Amérique Latine 2013/3(90), 39-56.

Vega Centeno, P., Belaúnde, P., Vilela, M., Fernández de Córdova, G., Moschella, P., Dammert, M., Pereyra, O, Bensus, V. \& Del Pozo, J. M. (2015). La densificación urbana en el siglo XXI como desafio para la planificación de Lima. Proyecto de investigación (20132015), Centro de Investigación de la Arquitectura y la Ciudad (CIAC)-Pontificia Universidad Católica del Perú (PUCP). 